\title{
Clinical characteristics and management of late urinary symptom flare following stereotactic body radiation therapy for prostate cancer
}

\section{Jennifer A. Woo ${ }^{1 \dagger}$, Leonard N. Chen ${ }^{1+}$, Aditi Bhagat ${ }^{1}$, Eric K. Oermann ${ }^{1}$, Joy S. Kim ${ }^{1}$, Rudy Moures ${ }^{1}$, Thomas Yung ${ }^{1}$, Siyuan Lei ${ }^{1}$, Brian T. Collins ${ }^{1}$, Deepak Kumar ${ }^{2}$, Simeng Suy ${ }^{1}$, Anatoly Dritschilo ${ }^{1}$, John H. Lynch ${ }^{3}$ and Sean P. Collins ${ }^{1}$ *}

1 Department of Radiation Medicine, Georgetown University Hospital, Washington, DC, USA

${ }^{2}$ Cancer Research Laboratory, Department of Biology, University of the District of Columbia, Washington, DC, USA

${ }^{3}$ Department of Urology, Georgetown University Hospital, Washington, DC, USA

\section{Edited by:}

Alan Jay Katz, Flushing Radiation

Oncology, USA

\section{Reviewed by:}

Daniel Grant Petereit, Rapid City Regional Hospital, USA

Michael T. Milano, University of

Rochester, USA

${ }^{*}$ Correspondence:

Sean P. Collins, Department of Radiation Medicine, Georgetown University Medical Center, 3800 Reservoir Road, N.W., Washington, DC 20007, USA

e-mail: SPC9@gunet.georgetown.edu

${ }^{\dagger}$ Jennifer A. Woo and Leonard N. Chen have contributed equally to this work.
Purpose: Stereotactic body radiation therapy (SBRT) is increasingly utilized as primary treatment for clinically localized prostate cancer. While acute post-SBRT urinary symptoms are well recognized, the late genitourinary toxicity of SBRT has not been fully described. Here, we characterize the clinical features of late urinary symptom flare and recommend conservative symptom management approaches that may alleviate the associated bother.

Methods: Between February 2008 and August 2011, 216 men with clinically localized prostate cancer were treated definitively with SBRT at Georgetown University Hospital. Treatment was delivered using the CyberKnife with doses of 35-36.25 Gy in five fractions. The prevalence of each of five Common Terminology Criteria for Adverse Events (CTCAE) graded urinary toxicities was assessed at each follow-up visit. Medication usage was documented at each visit. Patient-reported urinary symptoms were assessed using the American Urological Association (AUA) symptom score and the Expanded Prostate Cancer Index Composite (EPIC)-26 at 1, 3, 6, 9, 12, 18, and 24 months. Late urinary symptom flare was defined as an increase in the AUA symptom score of $\geq 5$ points above baseline with a degree of severity in the moderate to severe range (AUA symptom score $\geq 15$ ). The relationship between the occurrence of flare and pre-treatment characteristics were examined.

Results: For all patients, the AUA symptom score spiked transiently at 1 month post-SBRT. Of the 216 patients, 29 (13.4\%) experienced a second transient increase in the AUA symptom score that met the criteria for late urinary symptom flare. Among flare patients, the median age was 66 years compared to 70 for those without flare $(p=0.007)$. In patients who experienced flare, CTCAE urinary toxicities including dysuria, frequency/urgency, and retention peaked at 9-18months, and alpha-antagonist utilization increased at 1 month post-treatment, rose sharply at 12 months post-treatment, and peaked at 18 months (85\%) before decreasing at 24 months. The EPIC urinary summary score of flare patients declined transiently at 1 month and experienced a second, more protracted decline between 6 and 18 months before returning to near baseline at 2-year post-SBRT. Statistically and clinically significant increases in patient-reported frequency, weak stream, and dysuria were seen at 12 months post-SBRT. Among flare patients, $42.9 \%$ felt that urination was a moderate to big problem at 12 months following SBRT.

Conclusion: In this study, we characterize late urinary symptom flare following SBRT. Late urinary symptom flare is a constellation of symptoms including urinary frequency/urgency, weak stream, and dysuria that transiently occurs 6-18 months post-SBRT. Provision of appropriate anticipatory counseling and the maintenance of prophylactic alpha-antagonists may limit the bother associated with this syndrome.

Keywords: prostate cancer, SBRT, CyberKnife, EPIC, AUA symptom score, genitourinary toxicity, late urinary symptom flare, bother 


\section{INTRODUCTION}

Due to unavoidable dose to the bladder neck and prostatic urethra, genitourinary $(\mathrm{GU})$ toxicities are common following prostate cancer radiotherapy $(1,2)$. After external beam radiation therapy, incidence of late GU toxicity ( $\geq$ grade 2 ) ranges from 10 to $30 \%$ (3-5). Previous studies have recognized older age (6), higher radiation dose (5), and prior transurethral resection of the prostate (TURP) (7) as risk factors for late GU toxicity following external beam radiotherapy. GU toxicities are rarely an isolated finding, occurring more commonly as a complex of lower urinary tract symptoms (LUTS) (8). Recent data suggest that many of these toxicities may resolve with time (9) and analysis of actuarial incidence may over-estimate their clinical significance (10).

Patient responses to validated questionnaires may better define the longitudinal pattern of GU toxicity following radiotherapy compared with physician-reported data (11). Late urinary symptom flare was first described when urinary symptom questionnaires were administered to patients following prostate brachytherapy (12-14) and later confirmed by others $(15,16)$. Younger age at time of implantation was the only pre-treatment characteristic consistently associated with an increased risk of flare.

A case of late urinary symptom flare following stereotactic body radiation therapy (SBRT) was first reported by Suy et al. (17). It occurred 1-year post-SBRT and the patient eventually underwent a transurethral resection of prostate tissue (TURP). Microscopic evaluation of the TURP specimen suggested an inflammatory process (17). Subsequently, we described a series of patients experiencing post-SBRT late urinary toxicity, which resolved with conservative treatment such as alpha-blockers and/or brief steroid tapers (8). Endoscopic evaluation of these patients revealed bladder neck/urethral hyperemia suggestive of cystourethritis.

While acute GU toxicity post-SBRT is well characterized (8, 18-24), the late GU toxicity of SBRT has not been fully described. Here, we characterize the clinical features of late urinary symptom flare following SBRT, and recommend conservative symptom management approaches that may alleviate bother and minimize unnecessary interventions such as cystoscopy.

\section{MATERIALS AND METHODS PATIENT SELECTION}

Patients eligible for study inclusion had histologically confirmed, clinically localized adenocarcinoma of the prostate treated per our institutional protocol. Patients eligible for inclusion in this study had a prostate-specific antigen (PSA) $<40 \mathrm{ng} / \mathrm{ml}$, clinical stage T1c-T2c, and a Gleason score of $\leq 8$. Exclusion criteria included clinically involved lymph nodes on imaging, distant metastases on bone scan; prior pelvic radiotherapy and/or prior radical prostate surgery. Institutional IRB approval was obtained for retrospective review of data that was prospectively collected in our institutional database.

\section{TREATMENT PLANNING AND DELIVERY}

Stereotactic body radiation therapy treatment planning and delivery were conducted as previously described $(25,26)$. Briefly, gold fiducials were placed into the prostate. Fused CT and MR images were used for treatment planning. The clinical target volume (CTV) included the prostate and the proximal seminal vesicles. The planning target volume (PTV) equaled the CTV expanded $3 \mathrm{~mm}$ posteriorly and $5 \mathrm{~mm}$ in all other dimensions. The prescription dose was $35-36.25$ Gy to the PTV delivered in five fractions of 7-7.25 Gy over 1-2 weeks (27). The prescription isodose line was limited to $\geq 75 \%$. The bladder and membranous urethra were contoured and evaluated with dose-volume histogram analysis during treatment planning using Multiplan (Accuray Inc., Sunnyvale, CA, USA) inverse treatment planning. Critical structure dose constraints were as previously described (26). To minimize the risk of local recurrence, no attempt was made to limit the dose to the prostatic urethra. Target position was verified during treatment using paired, orthogonal X-ray images $(25,28)$.

\section{FOLLOW-UP AND STATISTICAL ANALYSIS}

Prostate-specific antigen levels were obtained before treatment, 1 month after the completion of SBRT, and during routine followup visits every 3 months for the first year and every 6 months for the second year of follow-up. All medications, including alpha-antagonists, corticosteroids, non-steroidal antiinflammatory drugs (NSAIDs), and urethral analgesics were documented at each visit. Toxicity was assessed prospectively at follow-up visits using the National Cancer Institute (NCI) Common Terminology Criteria for Adverse Events (CTCAE) version 3.0. Patient-reported outcomes were assessed pre-treatment and at follow-up visits using the American Urological Association (AUA) symptom score (29) and the Expanded Prostate Cancer Index Composite (EPIC) short form (30).

Student's $t$-test was used to assess differences in ongoing PSA levels. Wilcoxon signed rank test and Mann-Whitney $U$ test were used to analyze the quality of life scores in comparison to baseline. Sample medians and ranges were used to describe PSA levels. A clinically significant urinary flare was prospectively defined as an AUA symptom score $\geq 15$ with an increase of $\geq 5$ points above baseline $(15,16)$. Based upon published results, a benign PSA bounce was defined as a PSA rise of $0.2 \mathrm{ng} / \mathrm{ml}$ or more above its previous nadir with a subsequent decline to that nadir or lower (31). To statistically compare changes between time points, the levels of responses were assigned a score and the significance of the mean changes in the scores was assessed by paired $t$-test. EPIC scores for the urinary domain and its individual questions range from 0 to 100 with lower values representing worsening urinary symptoms. The minimally important difference (MID) in EPIC score was defined as a change of one-half standard deviation (SD) from the baseline (32).

The impact of baseline patient characteristics on the incidence of late urinary symptom flare was evaluated by univariate and multivariate analyses. Univariate analysis of variance (ANOVA) was used to detect significant relationship between patient characteristics and urinary symptom flare. In multivariate analysis, stepwise ordinal logistic regression modeling was used to determine independent factors predicting late urinary symptom flare. The baseline patient characteristics that were included as variables in the univariate and multivariate analyses included age, race, D'Amico Risk Group, Gleason score, T-Stage, prostate volume, PSA, testosterone level, baseline AUA symptom score, alphaantagonist usage, baseline EPIC urinary summary score, partner 
status, work status, Charlson comorbidity index, prior procedure for benign prostatic hyperplasia (BPH), and pre-treatment androgen deprivation therapy (ADT). All tests were two-tailed, and a $p$-value $<0.05$ was considered significant. IBM ${ }^{\circledR}$ SPSS version 21 and MedCalc ${ }^{\circledR}$ version 12.6.1.0 were used to perform the statistical analyses.

\section{RESULTS}

From August 2008 to August 2011, 216 patients with clinically localized prostate adenocarcinoma were treated per our institutional SBRT monotherapy protocol. The patients were followed for a minimum of 24 months following SBRT. The median patient age was 69 years (48-90 years) (Table 1). The percentage of patients self-identified as white was 56.5 and $37.5 \%$ as black. Comorbidities were common. The median prostate volume was $38 \mathrm{cc}$ (11.6$138.7 \mathrm{cc}$ ) and $8.8 \%$ had prior procedures for $\mathrm{BPH}$. There were $38.4 \%$ low-risk patients, $51.4 \%$ patients were intermediate-risk, and $10.2 \%$ patients were high-risk. Twenty-nine patients (13.4\%) also received ADT. A dose of 36.25 Gy delivered in five 7.25 Gy fractions was used for $87.5 \%$ of patients.

The mean AUA symptom score increased transiently at 1 month post-SBRT and returned to near baseline by 3 months post-SBRT (Figure 1A). This acute increase was both statistically $(p<0.0001)$ and clinically significant $(\mathrm{MID}=2.99)$. A second late protracted increase occurred between 9 and 18 months (Figure 1A). Transient late urinary symptom flare (occurring $\geq 6$ months after completing treatment) occurred in $13.4 \%$ of the patients (Figure 1B). The median flare magnitude was 13 and the median time to flare was 9 months (range, 6-18 months) (Figure 1C). The mean AUA score reduced to below baseline at 2 years post-SBRT. Among flare patients, the median age was 66 years compared to 70 for those without flare $(p=0.007)$ (Table 2$)$. No other baseline patient characteristics were significantly associated with flare following SBRT.

A PSA bounce of $0.2 \mathrm{ng} / \mathrm{ml}$ occurred in $30.1 \%$ of the study group of 216 patients. A total of $9 / 29$ patients $(31.0 \%)$ of the

Table 1 | Patient demographics and characteristics.

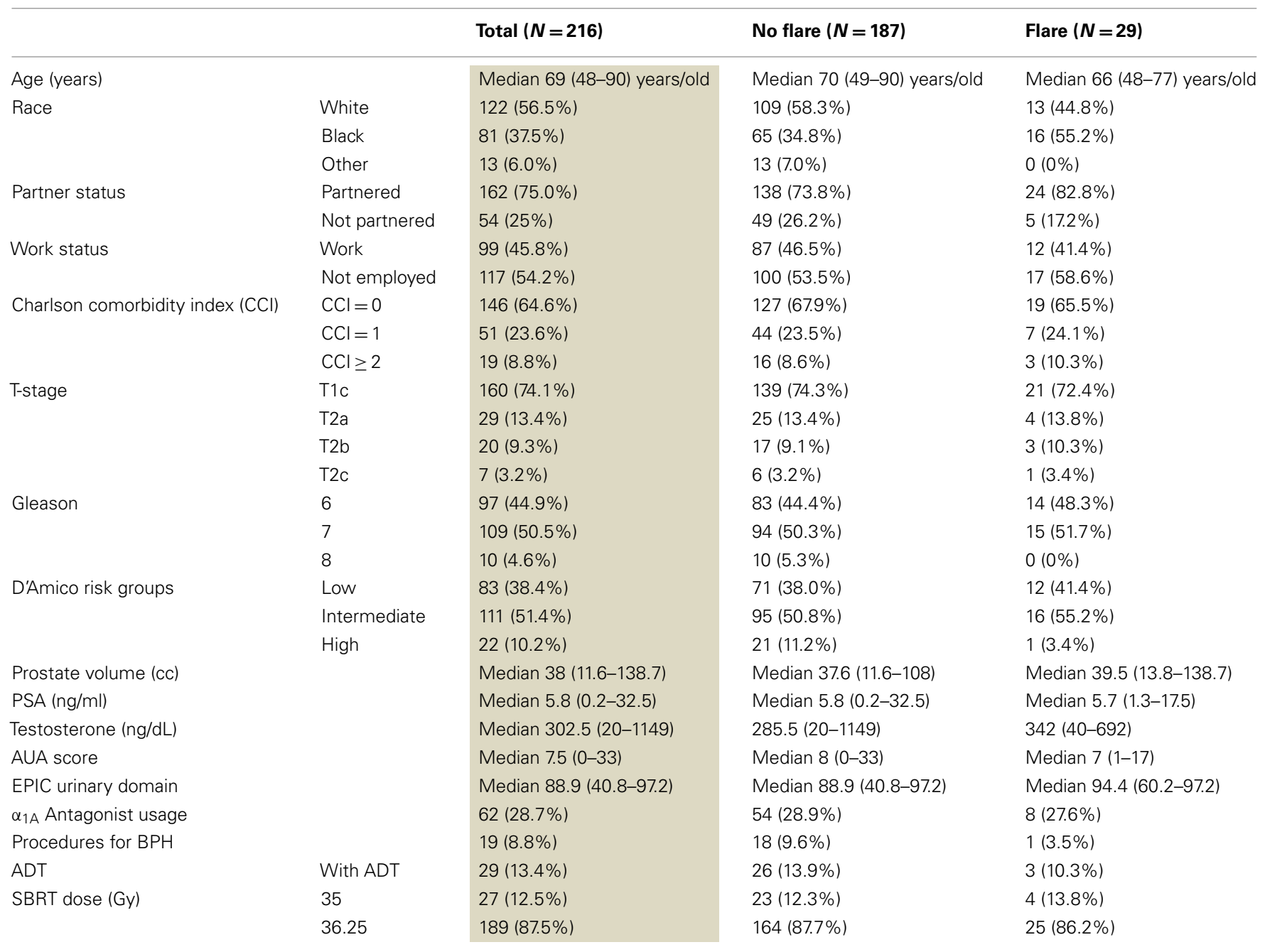

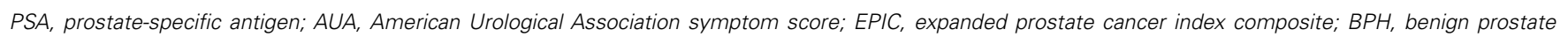
hyperplasia; ADT, androgen deprivation therapy; SBRT, stereotactic body radiation therapy. 

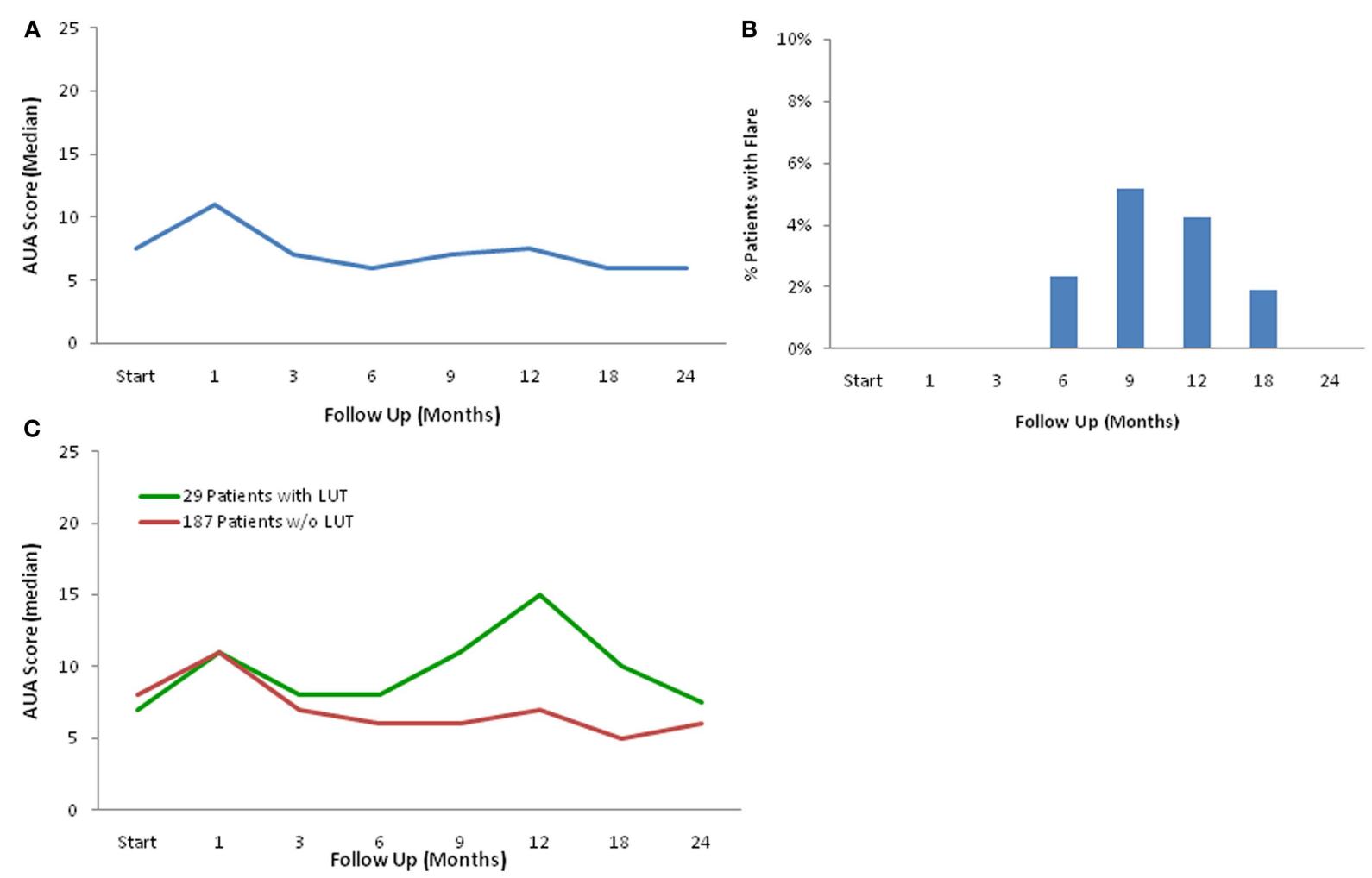

FIGURE 1 | Late urinary symptom flare. (A) AUA symptom score at baseline and following SBRT for prostate cancer. (B) Percentage of patients with urinary symptom flare at each follow-up. (C) AUA symptom score in patients with and without late urinary symptom flare. Thresholds for clinically significant changes in scores (1/2 standard deviation above and below the baseline). AUA scores range from 0 to 35 with higher values representing worsening urinary symptoms. flare patients experienced a PSA bounce compared with 56/187 $(29.9 \%)$ of the non-flare patients who experienced a bounce. Of the nine patients who experienced both a late urinary symptom flare and benign PSA bounce, two experienced the bounce and flare on the same follow-up evaluation. In three patients, the PSA bounce occurred before the flare, and in four patients the bounce occurred after the symptom flare.

For patients who experienced late urinary symptom flare, the prevalence of each of five CTCAE-graded urinary toxicities (hematuria, dysuria, incontinence, frequency, urgency, and retention) during the first 2 years of follow-up is illustrated in Table 3. Dysuria, urinary frequency/urgency, and retention were the most commonly experienced late toxicities. At baseline and each follow-up, usage of medication for urinary symptoms was assessed (Table 4; Figure 2). At baseline, 31\% of patients reported using alpha-antagonists; this increased at 1 month posttreatment, rose sharply at 12 months post-treatment, and peaked at 18 months $(85.2 \%)$ before decreasing at 24 months. Similarly, anti-inflammatory and urethral analgesic use peaked at 12 and 18 months, respectively.

The declines in the EPIC urinary scores paralleled the increases in the AUA symptom scores. The EPIC urinary summary score declined transiently at 1 month post-SBRT and returned to near baseline by 3 months post-SBRT (Figure 3A). This acute decline was both statistically $(p<0.0001)$ and clinically significant
( $\mathrm{MID}=6.48)$. A second late, protracted decline occurred between 9 and 18 months (Figure 3A). Transient late declines in the EPIC urinary summary domain were occurred almost exclusively in patients who experienced late urinary symptom flare (Figure 3B). Statistically and clinically significant increases in patient-reported frequency, weak stream, and dysuria were seen in flare patients at 12 months post-SBRT (Figure 4). The EPIC urinary summary score returned to near baseline by 2 years post-SBRT (Figures 3A,B and 4).

At baseline, $9.3 \%$ of the patients felt that urination was a moderate to big problem (Table 5). The mean EPIC urinary bother score was 78.2 at baseline (Figure 5A). Urinary bother increased following treatment with the mean score decreasing to 66.4 at 1 month post-treatment $(p<0.0001)$ (Figure 5A). However, only $17.5 \%$ of patients felt that urination was a moderate to big problem at 1 month following treatment (Table 5). Although urinary bother declined quickly, a second late increase in urinary bother was observed with the mean urinary bother score decreasing to 70.8 at 12 months $(p=0.009)$ (Figure 5A). Transient late declines in the EPIC urinary bother score were more common in patients who experienced late urinary symptom flare (Figure 5B). At 12 months post-treatment, $42.9 \%$ of the flare patients felt that urination was a moderate to big problem (Table 5). By 2 years following SBRT, urinary bother returned to near baseline in both flare and non-flare patients (Figure 5B). 


\section{DISCUSSION}

While conventionally fractionated intensity-modulated radiotherapy (IMRT) and brachytherapy remain the most commonly used radiation therapy modalities for clinically localized prostate cancer, SBRT is increasingly utilized as primary treatment for low

Table 2 | Impact of baseline patient characteristics on the incidence of late urinary symptom flare 2 years post-SBRT.

\begin{tabular}{ll}
\hline Factors & $\boldsymbol{p}$-Value \\
\hline Age & $0.007^{*}$ \\
Race & 0.505 \\
D'Amico risk groups & 0.381 \\
Gleason score (6, 7, $\geq 8)$ & 0.453 \\
T-stage (T1c, palpable) & 0.865 \\
Prostate volume & 0.820 \\
PSA & 0.299 \\
Testosterone level & 0.111 \\
Initial AUA & 0.080 \\
a1A antagonist usage & 0.975 \\
Initial EPIC urinary domain & 0.166 \\
Partner status & 0.812 \\
Work status & 0.599 \\
Charlson comorbidity index & 0.794 \\
Procedure for BPH & 0.212 \\
Androgen deprivation therapy & 0.282 \\
\hline
\end{tabular}

*Significant in multivariate analysis; ${ }^{*}$ significant in univariate analysis. PSA, prostate-specific antigen; AUA, American Urological Association urinary symptom score; EPIC, expanded prostate cancer index composite; $B P H$, benign prostate hypertrophy. to intermediate-risk disease. Data from several single-institution series $(8,20,22,33)$, multi-institutional phase I study (21), and a multi-institutional registry $(23,24)$ suggest SBRT offers high rates of biochemical control and low rates of grade 3 and higher toxicities, comparable with conventionally fractionated IMRT and brachytherapy. The maturation of these data is reflected in ASTRO's recent statement that SBRT can be "considered an appropriate alternative for select patients with low to intermediaterisk disease" (ASTRO Model Policies, 2012, https://www.astro.org/ Practice-Management/Reimbursement/Model-Policies.aspx). As utilization of SBRT for prostate cancer will likely increase in coming years, it is imperative to understand its impact on patients' quality of life.

Some studies have suggested an increased incidence of late GU toxicities following hypofractionated radiation therapy when compared to conventionally fractionated treatment (34-36). Our previous study showed a $30 \%$ incidence of late grade $2 \mathrm{GU}$ toxicities in the first 2 years following prostate SBRT (8). However, the majority of these patients experienced resolution of their GU toxicities with time. Reporting toxicities as actuarial incidence might overstate the significance of such problems $(10,37)$. In our opinion, prevalence better reflects the impact of a given toxicity on a patient's quality of life, and should be the standard approach for reporting toxicities.

Our analysis of the impact of baseline patient characteristics on the incidence of late urinary symptom flare revealed that young age was the only factor associated with an increased risk of developing flare. The association with age has been previously been seen with late urinary symptom flare following brachytherapy (14). To date, no mechanistic rationale has been determined. Surprisingly, pre-treatment urinary function was not significantly associated with flare in this study. Nor could we

Table 3 | Prevalence of CTCAE-graded urinary toxicities in flare patients following SBRT for prostate cancer.

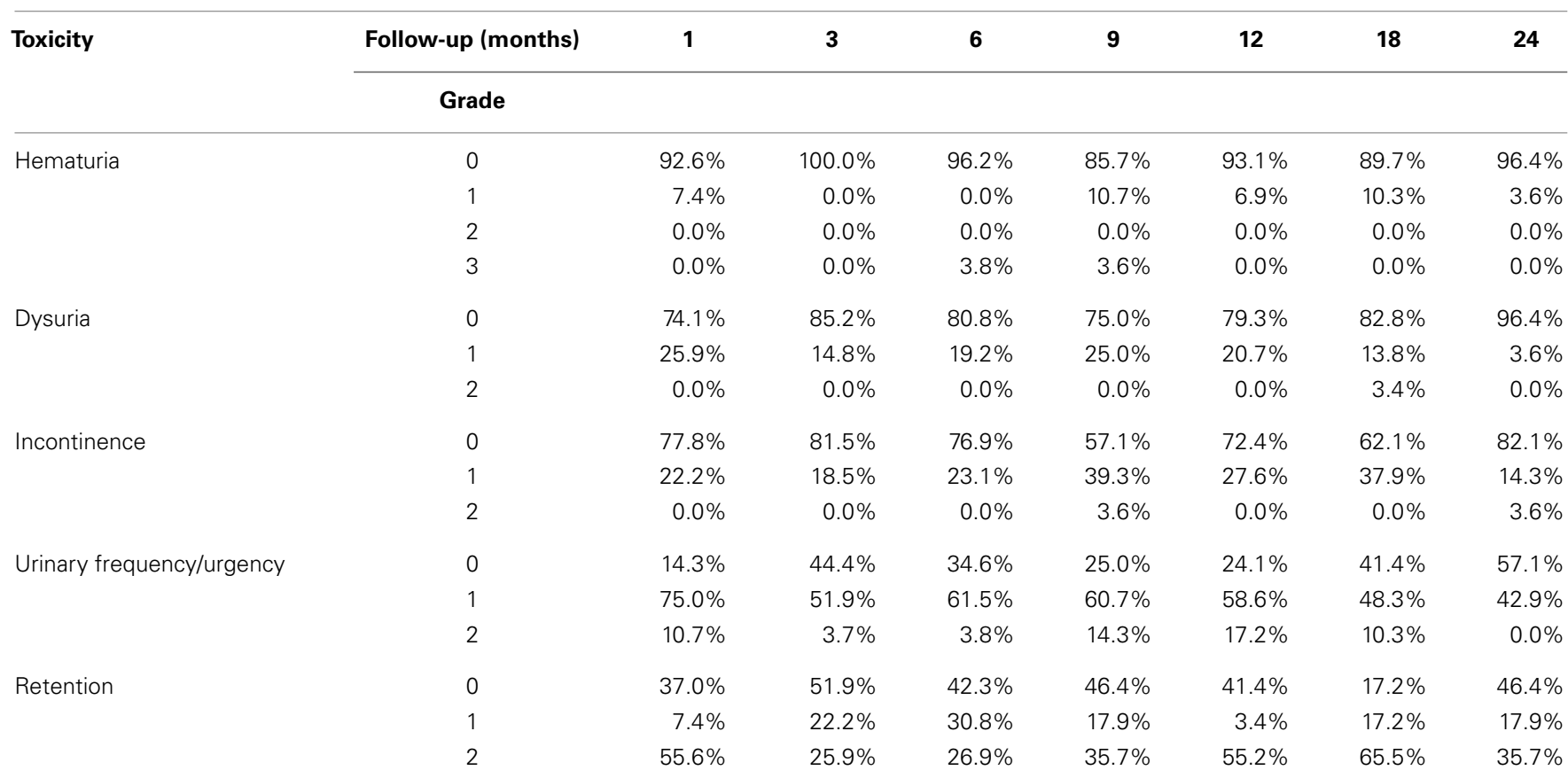


Table 4 | Percent of flare patients utilized medications for urinary symptom relief at baseline and following SBRT for prostate cancer.

\begin{tabular}{lrrrrrrrr}
\hline & Start & $\mathbf{1}$ & $\mathbf{3}$ & $\mathbf{6}$ & $\mathbf{9}$ & $\mathbf{1 2}$ & $\mathbf{1 8}$ \\
\hline$\alpha_{1 \text { A Inhibitor (\%) }}$ & 31.03 & 77.78 & 35.71 & 34.62 & 42.86 & 71.43 & 85.19 \\
Anti-inflammatory (\%) & 0.00 & 7.41 & 3.57 & 3.85 & 14.29 & 17.86 & 3.70 \\
Urethral analgesics (\%) & 0.00 & 0.00 & 0.00 & 0.00 & 3.57 & 3.57 & 3.70 & 0.00
\end{tabular}

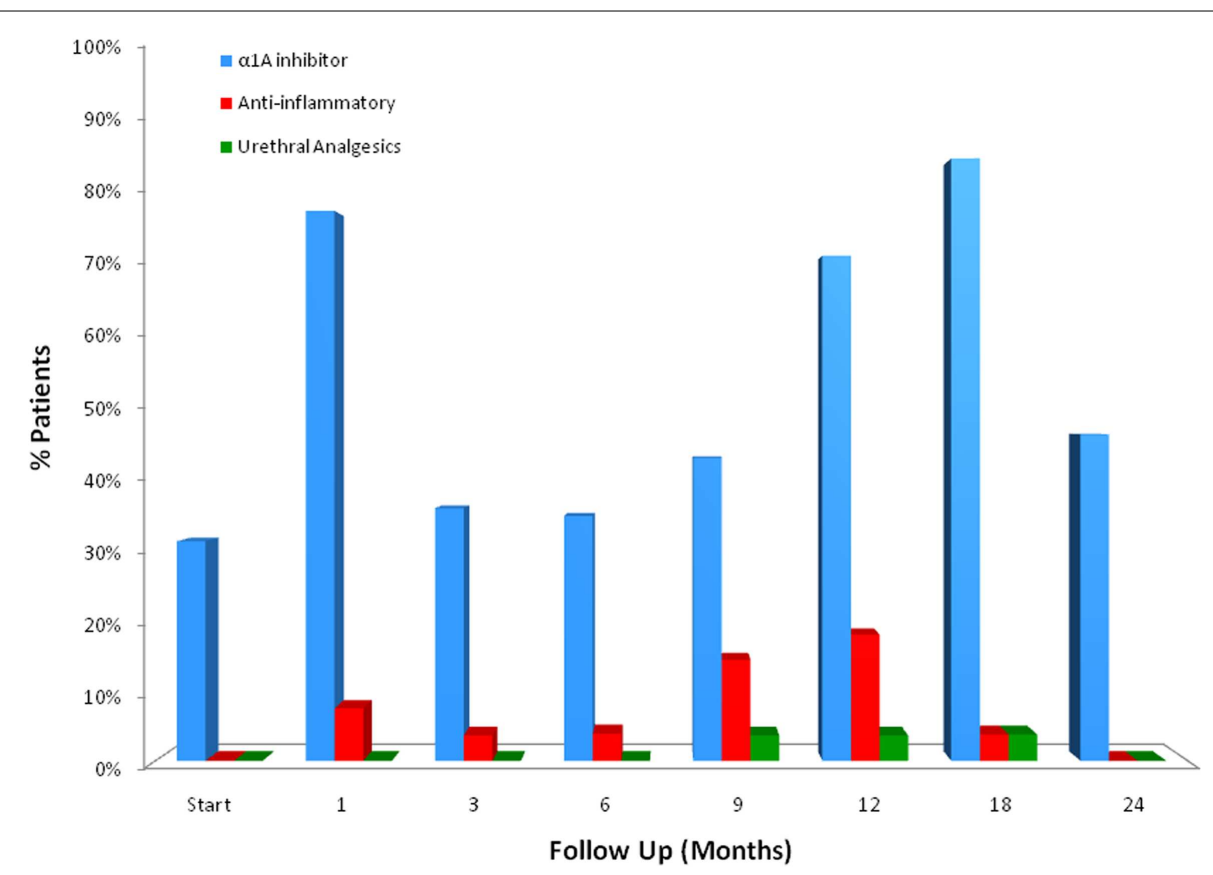

FIGURE 2 | Medication utilization for urinary symptom relief by flare patients at baseline and following SBRT for prostate cancer.
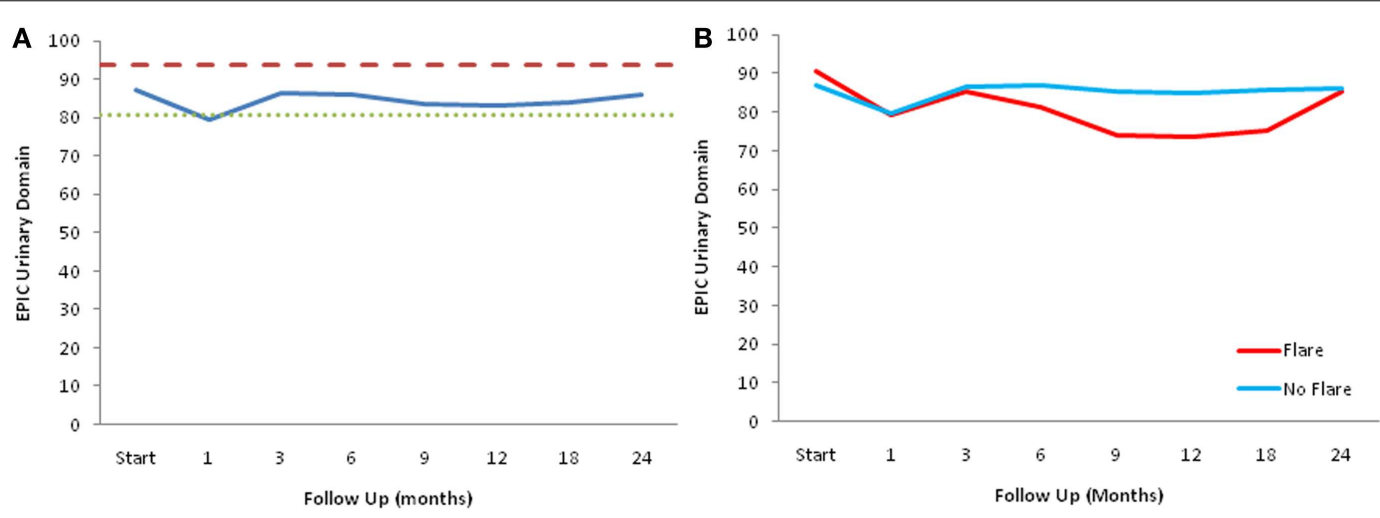

FIGURE 3 | EPIC urinary summary domain scores. (A) EPIC urinary summary domain scores at baseline and following SBRT for prostate cancer. (B) EPIC urinary summary domain scores in patients with and without late urinary symptom flare. Thresholds for clinically significant changes in scores $(1 / 2$ standard deviation above and below the baseline) are marked with dashed lines. EPIC scores range from 0 to 100 with higher values representing a more favorable health-related QOL.

establish a relationship between the severity of acute urinary symptoms and the incidence of late urinary symptom flare (data not shown) (38).
Endoscopic evaluation of these patients suggests that these symptoms may be caused by radiation-induced cystourethritis. An inflammatory etiology is supported by late urinary symptom 


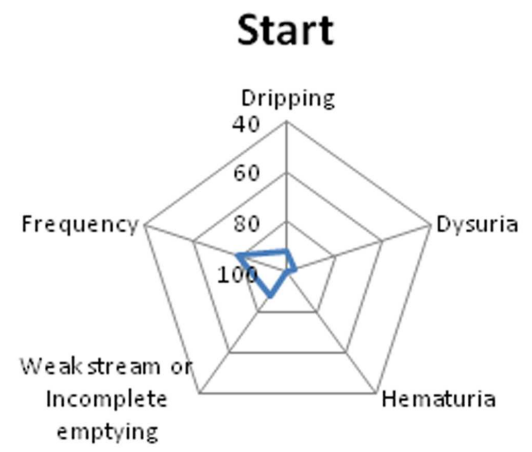

12 Mon

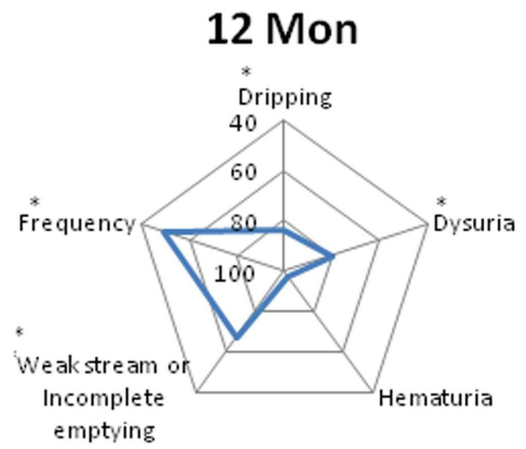

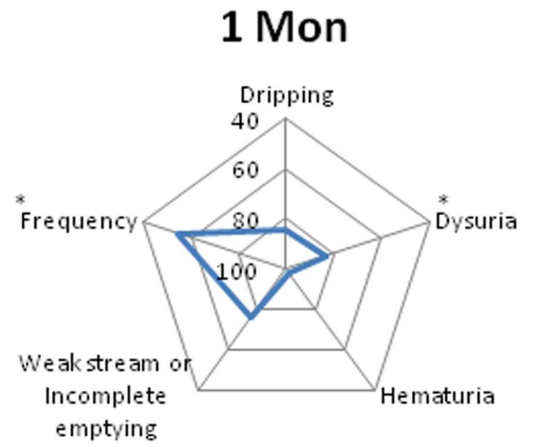

18 Mon

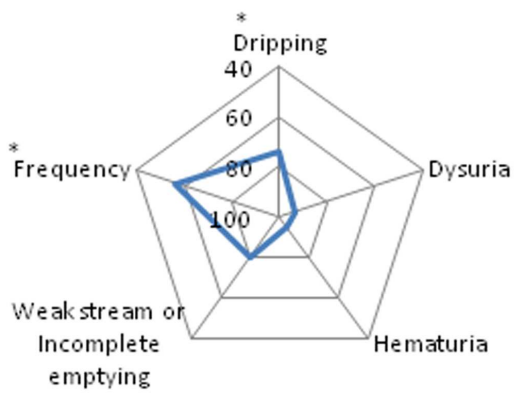

6 Mon

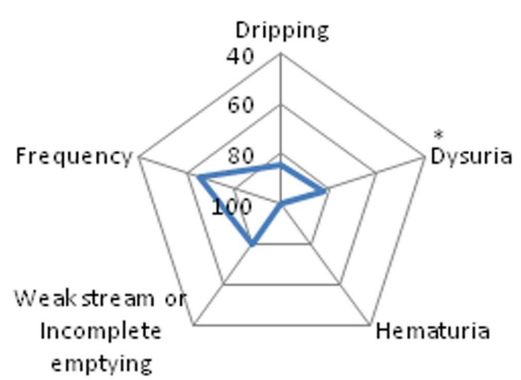

\section{Mon}

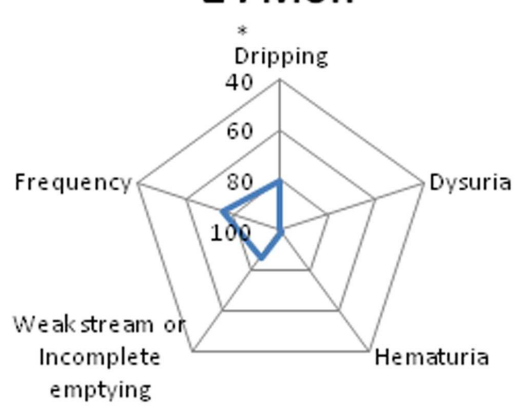

FIGURE 4 | Spider plots of individual EPIC urinary symptom scores at baseline and following SBRT for prostate cancer. Dripping-question 4A of the EPIC-26; dysuria-question 4B of the EPIC-26; hematuria-question $4 \mathrm{C}$ of the EPIC-26; weak stream or incomplete emptying-question 4D of the
EPIC-26; and frequency-question 4E of the EPIC-26. EPIC scores range from 0 to 100 with higher values representing a more favorable health-related $\mathrm{QOL}$. Changes in scores that are both statistically and clinically significant are marked with an asterisk $\left({ }^{*}\right)$.

Table 5 | Urinary bother in patients with and without late urinary symptom flare following SBRT for prostate cancer (patient-reported responses to question 5 of the EPIC-26).

\begin{tabular}{|c|c|c|c|c|c|c|c|c|}
\hline & Start & 1 Month & 3 Months & 6 Months & 9 Months & 12 Months & 18 Months & 24 Months \\
\hline \multicolumn{9}{|l|}{ ALL PATIENTS } \\
\hline N & 214 & 211 & 208 & 198 & 197 & 196 & 182 & 196 \\
\hline Very small-small problem (\%) & 44.4 & 57.8 & 50.5 & 48.0 & 44.2 & 49.5 & 41.8 & 45.9 \\
\hline Moderate-big problem (\%) & 9.3 & 17.5 & 8.7 & 9.1 & 15.7 & 14.8 & 13.7 & 8.7 \\
\hline$N$ & 185 & 184 & 180 & 172 & 169 & 168 & 155 & 170 \\
\hline No problem (\%) & 43.8 & 24.5 & 42.2 & 44.8 & 43.2 & 38.1 & 46.5 & 45.9 \\
\hline Very small-small problem (\%) & 47.6 & 58.2 & 48.9 & 46.5 & 43.2 & 51.8 & 41.3 & 45.9 \\
\hline Moderate-big problem (\%) & 8.6 & 17.4 & 8.9 & 8.7 & 13.6 & 10.1 & 12.3 & 8.2 \\
\hline$p$-Value & & $<0.0001 *$ & 0.7298 & 0.9282 & 0.5778 & 0.3185 & 0.6496 & 0.3608 \\
\hline Moderate-big problem (\%) & 13.8 & 18.5 & 7.1 & 11.5 & 28.6 & 42.9 & 22.2 & 11.5 \\
\hline$p$-Value & & $0.0483^{*}$ & 0.5614 & 0.4307 & $0.0056^{*}$ & $0.0008^{*}$ & 0.0737 & 0.3028 \\
\hline
\end{tabular}

*Changes in survey scores that are both statistically and clinically significant. 


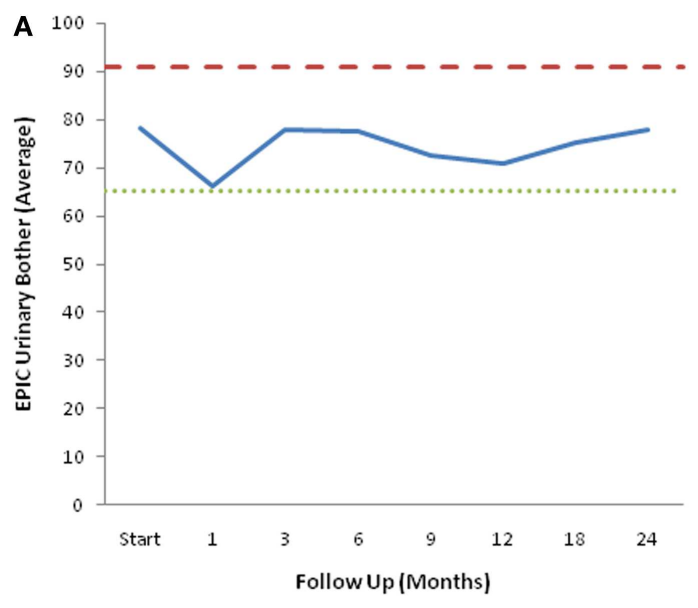

FIGURE 5 | EPIC urinary bother scores. (A) EPIC urinary bother scores at baseline and following SBRT for prostate cancer. (B) EPIC urinary bother scores in patients with and without late urinary symptom flare. Thresholds for

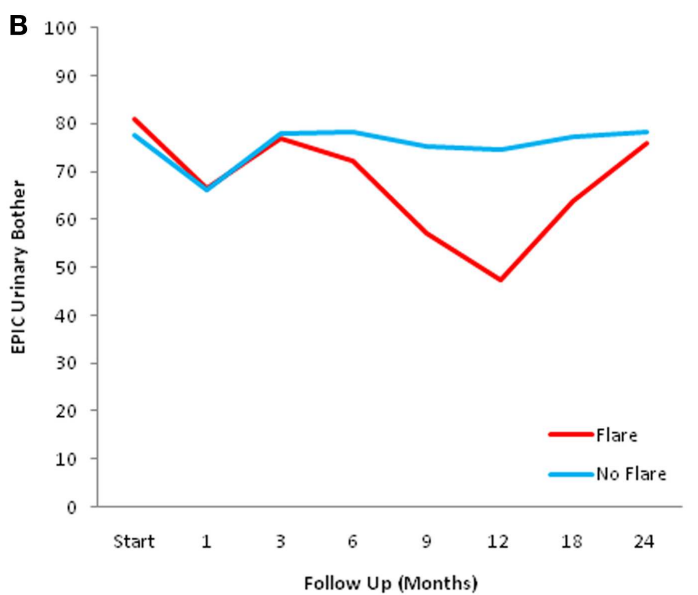

clinically significant changes in scores (1/2 standard deviation above and below the baseline) are marked with dashed lines. EPIC scores range from 0 to 100 with higher values representing a more favorable health-related $\mathrm{QOL}$. flare's delayed occurrence and rapid symptomatic response to oral steroids, not unlike post-radiation pneumonitis $(39,40)$. Radiation-induced inflammation is known to be dependent on radiation fractionation and the volume of the target organ irradiated $(41,42)$. Future studies should evaluate the impact of treatment factors (such as bladder neck/urethral dose) on the incidence of late urinary symptom flare (43). Interestingly, there was no temporal relationship between late urinary symptom flare and benign PSA bounces, suggesting a non-inflammatory nature for post-SBRT PSA bounces (14).

Similar to BPH-associated LUTS, patients with late urinary symptom flare present with obstructive and irritative voiding symptoms. Alpha-blockers have been shown to improve urinary flow and acute urinary symptoms following prostate radiotherapy $(44,45)$. In this series, most flare patients responded to alpha-antagonists. For those that did not respond adequately, we initiated a short oral corticosteroid taper (dexamethasome $4 \mathrm{mg}$ daily for 1 week followed by $2 \mathrm{mg}$ for 1 week, then discontinue) to reduce inflammation. With these conservative medical approaches, the majority of patients with urinary symptom flare recover to near baseline without the need for invasive procedures. Only a few patients required urethral anesthetics for persistent dysuria. Over the course of longer follow-up, we have not observed flare recurrences beyond 2 years since treatment (data not shown).

Prophylactic alpha-blockers have been shown to reduce urinary morbidity following prostate brachytherapy $(46,47)$. To maximize patient comfort, it is currently our institutional policy to initiate prophylactic alpha-antagonist use prior to initiating treatment. Patients are encouraged to continue alpha-antagonist usage until they return to their baseline urinary status and to reinitiate alpha-antagonists with any worsening of their urinary function.

We analyzed quality of life questionnaires to determine the degree of bother experienced by patients with late urinary symptom flare. Our results suggest that late urinary flare was highly bothersome to a significant percentage of the patients who experienced it. It is likely that these patients, not anticipating the onset of late urinary symptoms following a transient period of acute symptoms and a symptom-free interval, were particularly sensitive to perceived changes in their urinary status as they progressed further from the completion of SBRT (48). Clinicians should anticipate the possibility of late urinary symptoms and communicate to patients that a late transient flare may occur; this will better prepare patients for the onset of symptoms and potentially mitigate the perceived severity of the flare (48).

\section{CONCLUSION}

Our data provide compelling evidence for a clinical syndrome of transient late-onset urinary symptoms associated with SBRT for localized prostate cancer. The majority of flares occurs at 918 months post-SBRT and resolve by 24 months after treatment. Currently, it is difficult to predict which patients are at the highest risk of late urinary symptom flare. Provision of appropriate anticipatory counseling for all patients prior to treatment and utilization of alpha-antagonists may limit the need for invasive interventions.

\section{REFERENCES}

1. Bekelman JE, Mitra N, Efstathiou J, Liao K, Sunderland R, Yeboa DN, et al. Outcomes after intensity-modulated versus conformal radiotherapy in older men with nonmetastatic prostate cancer. Int J Radiat Oncol Biol Phys (2011) 81:e325-34. doi:10.1016/j.ijrobp.2011.02.006

2. Michalski JM, Yan Y, Watkins-Bruner D, Bosch WR, Winter K, Galvin JM, et al. Preliminary toxicity analysis of 3-dimensional conformal radiation therapy versus intensity modulated radiation therapy on the high-dose arm of the Radiation Therapy Oncology Group 0126 prostate cancer trial. Int J Radiat Oncol Biol Phys (2013) 87:932-8. doi:10.1016/j.ijrobp.2013.07.041

3. Peeters ST, Heemsbergen WD, Koper PC, Van Putten WL, Slot A, Dielwart MF, et al. Dose-response in radiotherapy for localized prostate cancer: results of the Dutch multicenter randomized phase III trial comparing 68 Gy of radiotherapy with 78 Gy. J Clin Oncol (2006) 24:1990-6. doi:10.1200/JCO.2005.05.2530

4. Kuban DA, Tucker SL, Dong L, Starkschall G, Huang EH, Cheung MR, et al. Long-term results of the M. D. Anderson randomized dose-escalation trial for 
prostate cancer. Int J Radiat Oncol Biol Phys (2008) 70:67-74. doi:10.1016/j. ijrobp.2007.06.054

5. Zelefsky MJ, Levin EJ, Hunt M, Yamada Y, Shippy AM, Jackson A, et al. Incidence of late rectal and urinary toxicities after three-dimensional conformal radiotherapy and intensity-modulated radiotherapy for localized prostate cancer. Int J Radiat Oncol Biol Phys (2008) 70:1124-9. doi:10.1016/j.ijrobp.2007.11.044

6. Ahmed AA, Egleston B, Alcantara P, Li L, Pollack A, Horwitz EM, et al. A novel method for predicting late genitourinary toxicity after prostate radiation therapy and the need for age-based risk-adapted dose constraints. Int J Radiat Oncol Biol Phys (2013) 86:709-15. doi:10.1016/j.ijrobp.2013.03.020

7. Devisetty K, Zorn KC, Katz MH, Jani AB, Liauw SL. External beam radiation therapy after transurethral resection of the prostate: a report on acute and late genitourinary toxicity. Int J Radiat Oncol Biol Phys (2010) 77:1060-5. doi:10.1016/j.ijrobp.2009.06.078

8. Chen LN, Suy S, Uhm S, Oermann EK, Ju AW, Chen V, et al. Stereotactic Body Radiation Therapy (SBRT) for clinically localized prostate cancer: the Georgetown University experience. Radiat Oncol (2013) 8:58. doi:10.1186/1748-717X8- 58

9. Schmid MP, Potter R, Bombosch V, Sljivic S, Kirisits C, Dorr W, et al. Late gastrointestinal and urogenital side-effects after radiotherapy - incidence and prevalence. Subgroup-analysis within the prospective Austrian-German phase II multicenter trial for localized prostate cancer. Radiother Oncol (2012) 104:114-8. doi:10.1016/j.radonc.2012.05.007

10. Peters LJ, Withers HR, Brown BW. Complicating issues in complication reporting. Int J Radiat Oncol Biol Phys (1995) 31:1349-51. doi:10.1016/0360-3016(95) 00041-V

11. Sonn GA, Sadetsky N, Presti JC, Litwin MS. Differing perceptions of quality of life in patients with prostate cancer and their doctors. J Urol (2013) 189:S59-65. doi:10.1016/j.juro.2012.11.032

12. Gelblum DY, Potters L, Ashley R, Waldbaum R, Wang XH, Leibel S. Urinary morbidity following ultrasound-guided transperineal prostate seed implantation. Int J Radiat Oncol Biol Phys (1999) 45:59-67. doi:10.1016/S0360-3016(99) 00176-5

13. Merrick GS, Butler WM, Lief JH, Dorsey AT. Temporal resolution of urinary morbidity following prostate brachytherapy. Int J Radiat Oncol Biol Phys (2000) 47:121-8. doi:10.1016/S0360-3016(99)00525-8

14. Cesaretti JA, Stone NN, Stock RG. Urinary symptom flare following I-125 prostate brachytherapy. Int J Radiat Oncol Biol Phys (2003) 56:1085-92. doi:10.1016/S0360-3016(03)00210-4

15. Crook J, Fleshner N, Roberts C, Pond G. Long-term urinary sequelae following 125iodine prostate brachytherapy. J Urol (2008) 179:141-145; discussion 146. doi:10.1016/j.juro.2007.08.136

16. Keyes M, Miller S, Moravan V, Pickles T, Liu M, Spadinger I, et al. Urinary symptom flare in $712125 \mathrm{I}$ prostate brachytherapy patients: long-term follow-up. Int J Radiat Oncol Biol Phys (2009) 75:649-55. doi:10.1016/j.ijrobp.2008.11.043

17. Suy S, Oermann E, Hanscom H, Lei S, Vahdat S, Yu X, et al. Histopathologic effects of hypofractionated robotic radiation therapy on malignant and benign prostate tissue. Technol Cancer Res Treat (2010) 9:583-7. doi:10.7785/tcrt.2012. 500168

18. Litwin MS, Gore JL, Kwan L, Brandeis JM, Lee SP, Withers HR, et al. Quality of life after surgery, external beam irradiation, or brachytherapy for early-stage prostate cancer. Cancer (2007) 109:2239-47. doi:10.1002/cncr.22676

19. Freeman DE, King CR. Stereotactic body radiotherapy for low-risk prostate cancer: five-year outcomes. Radiat Oncol (2011) 6:3. doi:10.1186/1748-717X-6-3

20. King CR, Brooks JD, Gill H, Presti JC Jr. Long-term outcomes from a prospective trial of stereotactic body radiotherapy for low-risk prostate cancer. Int J Radiat Oncol Biol Phys (2011) 82:877-82. doi:10.1016/j.ijrobp.2010.11.054

21. McBride SM, Wong DS, Dombrowski JJ, Harkins B, Tapella P, Hanscom HN, et al. Hypofractionated stereotactic body radiotherapy in low-risk prostate adenocarcinoma: preliminary results of a multi-institutional phase 1 feasibility trial. Cancer (2012) 118:3681-90. doi:10.1002/cncr.26699

22. Katz AJ, Santoro M, Diblasio F, Ashley R. Stereotactic body radiotherapy for localized prostate cancer: disease control and quality of life at 6 years. Radiat Oncol (2013) 8:118. doi:10.1186/1748-717X-8-118

23. King CR, Collins S, Fuller D, Wang PC, Kupelian P, Steinberg M, et al. Healthrelated quality of life after stereotactic body radiation therapy for localized prostate cancer: results from a multi-institutional consortium of prospective trials. Int J Radiat Oncol Biol Phys (2013) 87:939-45. doi:10.1016/j.ijrobp.2013. 08.019

24. King CR, Freeman D, Kaplan I, Fuller D, Bolzicco G, Collins S, et al. Stereotactic body radiotherapy for localized prostate cancer: pooled analysis from a multiinstitutional consortium of prospective phase II trials. Radiother Oncol (2013) 109:217-21. doi:10.1016/j.radonc.2013.08.030

25. Lei S, Piel N, Oermann EK, Chen V, Ju AW, Dahal KN, et al. Six-dimensional correction of intra-fractional prostate motion with cyberknife stereotactic body radiation therapy. Front Oncol (2011) 1:48. doi:10.3389/fonc.2011.00048

26. Oermann EK, Suy S, Hanscom HN, Kim JS, Lei S, Yu X, et al. Low incidence of new biochemical and clinical hypogonadism following hypofractionated stereotactic body radiation therapy (SBRT) monotherapy for low- to intermediate-risk prostate cancer. J Hematol Oncol (2011) 4:12. doi:10.1186/1756-8722-4-12

27. Katz AJ, Santoro M, Ashley R, Diblasio F. Stereotactic body radiation therapy for low- and low-intermediate-risk prostate cancer: is there a dose effect? Front Oncol (2011) 1:49. doi:10.3389/fonc.2011.00049

28. Xie Y, Djajaputra D, King CR, Hossain S, Ma L, Xing L. Intrafractional motion of the prostate during hypofractionated radiotherapy. Int J Radiat Oncol Biol Phys (2008) 72:236-46. doi:10.1016/j.ijrobp.2008.04.051

29. Barry MJ, Fowler FJ Jr, O’Leary MP, Bruskewitz RC, Holtgrewe HL, Mebust WK, et al. The American Urological Association symptom index for benign prostatic hyperplasia. The Measurement Committee of the American Urological Association. J Urol (1992) 148:1549-1557; discussion 1564.

30. Wei JT, Dunn RL, Litwin MS, Sandler HM, Sanda MG. Development and validation of the expanded prostate cancer index composite (EPIC) for comprehensive assessment of health-related quality of life in men with prostate cancer. Urology (2000) 56:899-905. doi:10.1016/S0090-4295(00)00858-X

31. Ciezki JP, Reddy CA, Garcia J, Angermeier K, Ulchaker J, Mahadevan A, et al. PSA kinetics after prostate brachytherapy: PSA bounce phenomenon and its implications for PSA doubling time. Int J Radiat Oncol Biol Phys (2006) 64:512-7. doi:10.1016/j.ijrobp.2005.07.960

32. Norman GR, Sloan JA, Wyrwich KW. Interpretation of changes in health-related quality of life: the remarkable universality of half a standard deviation. Med Care (2003) 41:582-92. doi:10.1097/00005650-200305000-00007

33. Friedland JL, Freeman DE, Masterson-Mcgary ME, Spellberg DM. Stereotactic body radiotherapy: an emerging treatment approach for localized prostate cancer. Technol Cancer Res Treat (2009) 8:387-92.

34. Arcangeli G, Fowler J, Gomellini S, Arcangeli S, Saracino B, Petrongari MG, et al. Acute and late toxicity in a randomized trial of conventional versus hypofractionated three-dimensional conformal radiotherapy for prostate cancer. Int J Radiat Oncol Biol Phys (2011) 79:1013-21. doi:10.1016/j.ijrobp.2009. 12.045

35. Pollack A, Walker G, Horwitz EM, Price R, Feigenberg S, Konski AA, et al. Randomized trial of hypofractionated external-beam radiotherapy for prostate cancer. J Clin Oncol (2013) 31:3860-8. doi:10.1200/JCO.2013.51.1972

36. Yu JB, Cramer LD, Herrin J, Soulos PR, Potosky AL, Gross CP. Stereotactic body radiation therapy versus intensity-modulated radiation therapy for prostate cancer: comparison of toxicity. J Clin Oncol (2014) 32:1195-201. doi:10.1200/JCO.2013.53.8652

37. Ghadjar P, Jackson A, Spratt DE, Oh JH, Munck af Rosenschold P, Kollmeier $\mathrm{M}$, et al. Patterns and predictors of amelioration of genitourinary toxicity after high-dose intensity-modulated radiation therapy for localized prostate cancer: implications for defining postradiotherapy urinary toxicity. Eur Urol (2013) 64:931-8. doi:10.1016/j.eururo.2013.02.001

38. Dorr W, Hendry JH. Consequential late effects in normal tissues. Radiother Oncol (2001) 61:223-31. doi:10.1016/S0167-8140(01)00429-7

39. Movsas B, Raffin TA, Epstein AH, Link CJ Jr. Pulmonary radiation injury. Chest (1997) 111:1061-76. doi:10.1378/chest.111.4.1061

40. Graves PR, Siddiqui F, Anscher MS, Movsas B. Radiation pulmonary toxicity: from mechanisms to management. Semin Radiat Oncol (2010) 20:201-7. doi:10.1016/j.semradonc.2010.01.010

41. Mehta V. Radiation pneumonitis and pulmonary fibrosis in non-small-cell lung cancer: pulmonary function, prediction, and prevention. Int J Radiat Oncol Biol Phys (2005) 63:5-24. doi:10.1016/j.ijrobp.2005.03.047

42. Ghafoori P, Marks LB, Vujaskovic Z, Kelsey CR. Radiation-induced lung injury. Assessment, management, and prevention. Oncology (Williston Park) (2008) 22:37-47; discussion 52-33. 
43. Ghadjar P, Zelefsky MJ, Spratt DE, Munck af Rosenschold P, Oh JH, Hunt M, et al. Impact of dose to the bladder trigone on long-term urinary function after high-dose intensity modulated radiation therapy for localized prostate cancer. Int J Radiat Oncol Biol Phys (2014) 88:339-44. doi:10.1016/j.ijrobp.2013. 10.042

44. Prosnitz RG, Schneider L, Manola J, Rocha S, Loffredo M, Lopes L, et al. Tamsulosin palliates radiation-induced urethritis in patients with prostate cancer: results of a pilot study. Int J Radiat Oncol Biol Phys (1999) 45:563-6. doi:10.1016/S0360-3016(99)00246-1

45. Zelefsky MJ, Ginor RX, Fuks Z, Leibel SA. Efficacy of selective alpha-1 blocker therapy in the treatment of acute urinary symptoms during radiotherapy for localized prostate cancer. Int J Radiat Oncol Biol Phys (1999) 45:567-70. doi:10.1016/S0360-3016(99)00232-1

46. Merrick GS, Butler WM, Wallner KE, Lief JH, Galbreath RW. Prophylactic versus therapeutic alpha-blockers after permanent prostate brachytherapy. Urology (2002) 60:650-5. doi:10.1016/S0090-4295(02)01840-X

47. Elshaikh MA, Ulchaker JC, Reddy CA, Angermeier KW, Klein EA, Chehade N, et al. Prophylactic tamsulosin (Flomax) in patients undergoing prostate 125I brachytherapy for prostate carcinoma: final report of a double-blind placebocontrolled randomized study. Int J Radiat Oncol Biol Phys (2005) 62:164-9. doi:10.1016/j.ijrobp.2004.09.036

48. Symon Z, Daignault S, Symon R, Dunn RL, Sanda MG, Sandler HM. Measuring patients' expectations regarding health-related quality-of-life outcomes associated with prostate cancer surgery or radiotherapy. Urology (2006) 68:1224-9. doi:10.1016/j.urology.2006.08.1092

Conflict of Interest Statement: Dr. Sean Collins and Dr. Brian Collins are consultants for Accuray, Inc. The other co-authors declare that the research was conducted in the absence of any commercial or financial relationships that could be construed as a potential conflict of interest.

Received: 01 April 2014; accepted: 09 May 2014; published online: 26 May 2014.

Citation: Woo JA, Chen LN, Bhagat A, Oermann EK, Kim JS, Moures R, Yung T, Lei S, Collins BT, Kumar D, Suy S, Dritschilo A, Lynch JH and Collins SP (2014) Clinical characteristics and management of late urinary symptom flare following stereotactic body radiation therapy for prostate cancer. Front. Oncol. 4:122. doi: 10.3389/fonc.2014.00122

This article was submitted to Radiation Oncology, a section of the journal Frontiers in Oncology.

Copyright (C) 2014 Woo, Chen, Bhagat, Oermann, Kim, Moures, Yung, Lei, Collins, Kumar, Suy, Dritschilo, Lynch and Collins. This is an open-access article distributed under the terms of the Creative Commons Attribution License (CC BY). The use, distribution or reproduction in other forums is permitted, provided the original author(s) or licensor are credited and that the original publication in this journal is cited, in accordance with accepted academic practice. No use, distribution or reproduction is permitted which does not comply with these terms. 